\title{
Recent results on the nonmesonic weak decay of hypernuclei within a one-meson-exchange model.
}

\author{
A. Parreño*, A. Ramos \\ Departament d'Estructura i Constituents de la Matèria, Universitat de Barcelona, \\ Diagonal 647, E-08028 Barcelona, Spain \\ C. Bennhold \\ Center for Nuclear Studies and Department of Physics, The George Washington University, \\ Washington DC, 20052, USA
}

(November 3, 2018)

\begin{abstract}
We update [1] the results presented in Ref. [2] for the nonmesonic decay (NMD) of ${ }_{\Lambda}^{12} \mathrm{C}$ and ${ }_{\Lambda}^{5} \mathrm{He}$. We pay special attention to the role played by Final State Intreractions (FSI) on the decay observables. We follow a One-MesonExchange (OME) model which includes the exchange of the $\pi, \rho, K, K^{*}, \eta$ and $\omega$ mesons. We also present recent predictions for different observables concerning the decay of the doubly strange ${ }_{\Lambda \Lambda}^{6} \mathrm{He}$ hypernucleus [3].
\end{abstract}

\section{INTRODUCTION}

The $\Lambda$ particle, the lightest among the hyperons, decays in free space into nucleons and pions, with a lifetime of $\approx 2.632 \times 10^{-10}$ sec. This (mesonic) decay mode is dominant for the very light s-shell hypernuclei but, as the number of nucleons increase, it gets suppressed due to the low momentum of the outgoing nucleon, which gets Pauli blocked. Therefore, for hypernuclei with $A \simeq 5$ or larger, the decay is assumed to proceed mainly via the two-body reaction $\Lambda N \rightarrow N N$, the so-called nonmesonic decay (NMD) mode. Since at present the availability of stable hyperon beams is very limited, this NMD mode is the only source of information on the $|\Delta S|=1$ hyperon-nucleon (YN) interaction. Further, the decay of doubly-strange hypernuclei, as the decay of ${ }_{\Lambda \Lambda}^{6}$ He studied here, provides additional information due to novel hyperon-induced decay mechanisms, namely $\Lambda \Lambda \rightarrow \Lambda N$ and $\Lambda \Lambda \rightarrow$ $\Sigma N$.

Our framework includes the exchange of the pseudoscalar $\pi, \eta, K$ octet for the long-range part while parametrizing the short-range part through the exchange of the vector $\rho, \omega$ and

\footnotetext{
*and IFAE, Universitat Autònoma de Barcelona, E-08193 Bellaterra, Barcelona, Spain.
} 
$K^{*}$ mesons. Realistic baryon-baryon forces for the $S=0,-1$ and -2 sectors [4] are used to account for the strong interaction in the initial and final states.

\section{FORMALISM}

Analytic expressions of the total and partial decay rates, as well as of the Parity Violating (PV) asymmetry for the decay of single- and double- $\Lambda$ hypernuclei can be found in Refs. [1.3]. Details on how to derive the transition potential and its final form can be found also there. Only some basic aspects of the formalism are going to be outlined here.

The transition potential is derived by performing a nonrelativistic reduction of the Feynman diagram associated to the exchange of the meson under consideration. In analogy to One-Boson-Exchange (OBE) based models of the strong interaction, the present formalism includes not only the exchange of the long-ranged pion, but also more massive mesons which account for shorter distances. Those are the $\rho, K, K^{*}, \eta$ and $\omega$ mesons. In order to account for finite size effects, we include a monopole form factor at each vertex, where the value of the cut-off depends on the meson.

As it is well known, one of the sources of uncertainty in OBE models comes from the coupling constants between baryons and mesons (BBM). In the strong sector the different interaction models use $\mathrm{SU}(3)$ in order to obtain the BBM couplings that are not constrained experimentally. Recently, the Nijmegen group has made available new baryon-baryon interactions in the strangeness $S=0,-1,-2,-3$ and -4 sectors [4], where the $S=-2 \rightarrow-4$ versions are $\mathrm{SU}(3)$ extensions of the models in the $S=0$ and -1 sectors, which are fitted to experimental data. The authors of Ref. [4] give six different models, which fit the available $N N$ and $Y N$ scattering data equally well but are characterized by different values of the magnetic vector $F /(F+D)$ ratio, ranging from 0.4447 (model NSC97a) to 0.3647 (model NSC97f).

In the weak sector, only the decay of the $\Lambda$ and $\Sigma$ hyperons into nucleons and pions can be experimentally observed. For the other mesons, $S U_{w}(6)$ represents a convenient tool to obtain the PV amplitudes, while for the Parity Conserving (PC) ones, we use a pole model [2,5] with only baryon pole resonances.

In order to take into account the effects of the strong interaction between the baryons, correlated wave functions are obtained from a $G$-matrix calculation for the initial $\Lambda \mathrm{N}$ and $\Lambda \Lambda$ states. Our treatment of FSI is restricted [1] to the study of the mutual influence between the two emitted baryons. To include the effect of these FSI in the decay process, we obtain a scattering BB wave function from a Lippmann-Schwinger ( $T$-matrix) equation using the NSC97f potential model of Ref. [4]. In the next section, we will also show the results obtained with other more simplistic approaches. These approaches include, for instance, the absence

of FSI or the use of a phenomenological correlation function multiplying the uncorrelated wave function.

\section{RESULTS}

We present updated results for the weak nonmesonic decay of hypernuclei to the light of the new Nijmegen baryon-baryon potentials [4]. These strong interaction models influence 
the weak decay mechanism, not only through the coupling constants and form factors at the strong vertex involved in the two-body reaction, $\Lambda N \rightarrow N N$ (and $\Lambda \Lambda \rightarrow Y N$ in $\Lambda \Lambda$ hypernuclei, where $Y$ denotes a hyperon in the final state), but also via the PC piece of the weak vertex, obtained from a pole model, as well as from the corresponding correlated wave functions for the initial $\Lambda N$ and final $N N$ states.

Table $\llbracket$ shows our estimations for the decay observables (in units of the free $\Lambda$ decay rate, $\Gamma_{\Lambda}=3.8 \times 10^{9} \mathrm{~s}^{-1}$ ) of ${ }_{\Lambda}^{5} \mathrm{He}$ and ${ }_{\Lambda}^{12} \mathrm{C}$. Those numbers have been obtained working consistently within each of the strong models of the Nijmegen group. The new results for the nonmesonic rates compare favourably with the present experimental data. The $\mathrm{n} / \mathrm{p}$ ratio has increased with respect to our previous works and it now lies practically within the lower side of the error band. The asymmetry for ${ }_{\Lambda}^{12} \mathrm{C}$ is also compatible with experiment [6] but that for ${ }_{\Lambda}^{5} \mathrm{He}$ disagrees strongly from the recent experimental observation [7]. The latter work finds a small and positive value for the elementary asymmetry parameter $a_{\Lambda}$ in ${ }_{\Lambda}^{5} \mathrm{He}$, while that for ${ }_{\Lambda}^{12} \mathrm{C}$ is large and negative. Our meson-exchange model does not explain the present experimental differences and understanding this issue is one of the current challenges, both experimental and theoretical, in the study of the weak decay of hypernuclei.

We have found a tremendous influence on the weak decay observables from the way FSI are considered, especially in the case of total and partial decay rates. In Table TI we compare the results obtained by using different approaches to implement FSI. A phenomenological implementation of FSI effects, $f_{\text {phen }}=1-j_{0}\left(q_{c} r\right)$ with $q_{c}=3.93 \mathrm{fm}^{-1}$, or not including them at all, gives rise to decay rates that differ by more than a factor of two, and to a neutronto-proton ratio about $20 \%$ larger from what is obtained with the more realistic calculation that uses the proper $N N$ scattering wave function. The $K$-matrix solution represents an approximation which is only appropriate for standing waves, i.e. non-propagating solutions, as is the case in the nuclear medium. The differences observed in the decay rates and the $\mathrm{n} / \mathrm{p}$ ratio are much larger than the uncertainties tied to the different strong interaction models commented above. Therefore, accurate calculations of the nonmesonic weak decay of hypernuclei demand a proper treatment of FSI effects through the solution of a $T$-matrix using realistic NN interactions.

Predictions for the decay observables of ${ }_{\Lambda \Lambda}^{6} \mathrm{He}$ are shown in Table [II]. The $\Lambda N \rightarrow N N$ rate is found to be more than twice as large as in ${ }_{\Lambda}^{5} \mathrm{He}$ due to the increased binding of the second $\Lambda$ hyperon. The total hyperon-induced rate is $4 \%$ of the total nonmesonic rate, and it is dominated by the $\Lambda \Lambda \rightarrow \Lambda n$ mode, which allows direct access to exotic vertices like $\Lambda \Lambda \mathrm{K}$, unencumbered by the usually dominant pion exchange. Indeed, one-loop log corrected $\chi \mathrm{PT}$ results [3] modify the $\Lambda \Lambda \rightarrow \Lambda n$ by $50 \%$ while changing the $\Lambda N \rightarrow N N$ only at the $15 \%$ level, demonstrating the power of this weak mechanism to test $\chi \mathrm{PT}$ in the weak $\mathrm{SU}(3)$ sector. With a free $\Lambda$ in the final state this new mode should be distinguishable from the usual nucleon-induced decay channels.

\footnotetext{
${ }^{1}$ We have to note here that this number could change in the light of the new data presented by Prof. Nakazawa during this conference: $B\left({ }_{\Lambda \Lambda}^{6} \mathrm{He}\right)=6.93 \pm 0.54 \mathrm{MeV}$ and $\Delta B\left({ }_{\Lambda \Lambda}^{6} \mathrm{He}\right)=0.69 \pm 0.54$ $\mathrm{MeV}$.
} 


\section{TABLES}

\begin{tabular}{|c|c|c|c|c|c|c|c|c|}
\hline & \multicolumn{2}{|c|}{$\overline{\Gamma_{n m}}$} & \multicolumn{2}{|c|}{$\overline{\overline{\Gamma_{n} / \Gamma_{p}}}$} & \multicolumn{2}{|c|}{$\overline{\Gamma_{p}}$} & \multicolumn{2}{|c|}{$\overline{A_{p}}$} \\
\hline & $\mathrm{a}$ & $\mathrm{f}$ & $\mathrm{a}$ & $\mathrm{f}$ & $\mathrm{a}$ & $f$ & $\mathrm{a}$ & $\mathrm{f}$ \\
\hline${ }_{\Lambda}^{5} \mathrm{He}$ & 0.425 & 0.317 & 0.343 & 0.457 & 0.317 & 0.218 & -0.675 & -0.682 \\
\hline EXP: & \multicolumn{2}{|c|}{$0.41 \pm 0.149$} & \multicolumn{2}{|c|}{$0.93 \pm 0.559$} & \multicolumn{2}{|c|}{$0.21 \pm 0.07$} & \multicolumn{2}{|c|}{$0.24 \pm 0.22$ [7] } \\
\hline$\overline{12} \mathrm{C}$ & 0.726 & 0.554 & 0.288 & 0.341 & 0.564 & 0.413 & 0.358 & 0.367 \\
\hline EXP: & $\begin{array}{r}1 \\
0.89= \\
1\end{array}$ & $\begin{array}{l}.08 \text { 8 } \\
\pm 0.03 \text { 10 } \\
0.2,9\end{array}$ & $\begin{array}{r}1 \\
1.87 \\
0 . \\
0.5\end{array}$ & 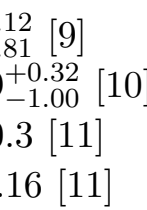 & $0.31^{-}$ & $\begin{array}{lll}8 & 10 \\
1 & \end{array}$ & $0.05 \pm$ & 0.53 己 \\
\hline
\end{tabular}

TABLE I. Weak decay observables for ${ }_{\Lambda}^{5} \mathrm{He}$ and ${ }_{\Lambda}^{12} \mathrm{C}$. The strong NSC97a (left column) and NSC97f (right column) potential models have been used [4]. For the final NN wave function we used the solution of a T-matrix equation with either NSC97a or NSC97f.

\begin{tabular}{lcccc}
\hline \hline$\overline{5} \mathrm{He}$ & $\Gamma_{n m}$ & $\Gamma_{n} / \Gamma_{p}$ & $\Gamma_{p}$ & $A_{p}$ \\
\hline$T$ & 0.317 & 0.457 & 0.218 & -0.682 \\
$K$ & 0.475 & 0.471 & 0.323 & -0.650 \\
$f_{\text {phen }}(r)$ & 0.766 & 0.619 & 0.473 & -0.671 \\
no FSI & 0.721 & 0.614 & 0.447 & -0.654 \\
\hline \hline
\end{tabular}

TABLE II. Weak decay observables for ${ }_{\Lambda}^{5} \mathrm{He}$ using different approaches to FSI. The NSC97f model has been used.

\begin{tabular}{lr|lr}
\hline \hline$\Lambda n \rightarrow n n$ & 0.30 & $\Lambda \Lambda \rightarrow \Lambda n$ & $3.6 \times 10^{-2}$ \\
$\Lambda p \rightarrow n p$ & 0.66 & $\Lambda \Lambda \rightarrow \Sigma^{0} n$ & $1.3 \times 10^{-3}$ \\
$\Lambda N \rightarrow N N$ & 0.96 & $\Lambda \Lambda \rightarrow \Sigma^{-} p$ & $2.6 \times 10^{-3}$ \\
\hline$\Gamma_{\mathrm{n}} / \Gamma_{\mathrm{p}}$ & 0.46 & $\Lambda \Lambda \rightarrow Y N$ & $4.0 \times 10^{-2}$ \\
\hline \hline
\end{tabular}

TABLE III. Partial weak decay rates for ${ }_{\Lambda \Lambda}^{6}$ He.

\section{ACKNOWLEDGMENTS}

This work has been partially supported by the U.S. Dept. of Energy under Grant No. DE-FG03-00-ER41132, by the DGICYT (Spain) under contract PB98-1247, by the Generalitat de Catalunya project SGR2000-24, and by the EEC-TMR Program EURODAPHNE under contract CT98-0169.

\footnotetext{
${ }^{2}$ This number has been obtained dividing the experimental asymmetry, $\mathcal{A}=-0.01 \pm 0.10$ [6], by a polarization of $P_{y}=-0.19$.
} 


\section{REFERENCES}

[1] A. Parreño and A. Ramos, E-print Archive: nucl-th/0104080.

[2] A. Parreño, A. Ramos, and C. Bennhold, Phys. Rev. C 56, 1997, p. 339.

[3] A. Parreño, A. Ramos, and C. Bennhold, E-print Archive: nucl-th/0106054.

[4] V.G.J. Stoks and Th.A. Rijken, Phys. Rev. C 59, 1999, p. 3009; Th.A. Rijken, V.G.J. Stoks and Y. Yamamoto, Phys. Rev. C 59, 1999, p. 21.

[5] J.F. Dubach, G.B. Feldman, B.R. Holstein, L. de la Torre, Ann. Phys. (N.Y.) 249, 1996, p. 146; L. de la Torre, Ph.D. Thesis, Univ. of Massachusetts, 1982.

[6] S. Ajimura et al., Phys. Lett. B282, 1992, p. 293.

[7] S. Ajimura et al., Phys. Rev. Lett. 18, 2000, p. 4052.

[8] H. Bhang et al., Phys. Rev. Lett. 81, 1998, p. 4321.

[9] J.J. Szymanski et al., Phys. Rev. C 43, 1991, p. 849.

[10] H. Noumi et al., Phys. Rev. C 52, 1995, p. 2936.

[11] A. Montwill et al., Nucl. Phys. A234, 1974, p. 413. 